\title{
Neutral Organic Super Electron Donors Made Catalytic
}

\author{
Simon Rohrbach, ${ }^{[a]}$ Rushabh S. Shah, ${ }^{[b]}$ Tell Tuttle, ${ }^{*[a]}$ and John A. Murphy ${ }^{*[a]}$
}

Abstract: Neutral organic super electron donors (SEDs) display impressive reducing power but, until now, it has not been possible to use them catalytically in radical chain reactions. This is because, following electron transfer, these donors form persistent radical cations that trap substrate-derived radicals. This paper unlocks a conceptually new approach to super electron donors that overcomes this issue, leading to the first catalytic neutral organic super electron donor.

Redox reactions occupy a central and rapidly developing role in organic chemistry. Organic electron donors have moved forward significantly since the reactions of TTF (tetrathiafulvalene) $\mathbf{1}^{[1]}$ and TDAE [tetrakis(diethylamino)ethene] 2 were explored, ${ }^{[2]}$ as witnessed in the reactivity of the increasingly powerful donors 3 6. ${ }^{[3,4]}$ TTF, $\mathbf{1}$, is a weak electron donor that can reduce arenediazonium salts, but not aryl halides. Upon oxidation, the $\pi$ system gains aromaticity as illustrated for structure $\mathbf{8}$ (Scheme 1). This aromatic driving force is a key determinant of electron donors reducing power. ${ }^{[4 a]}$ Stronger donors e.g. 2 use nitrogen lone pairs rather than sulfur lone pairs to stabilise radicals and cations in the oxidized forms. Combining the benefits of developing aromaticity and use of $\mathrm{N}$ atoms inspired the structural templates for neutral organic 'super electron donors' (SED) 3-6, which are defined as neutral ground state organic molecules that reduce aryl halides to aryl radicals or aryl anions. ${ }^{[4]}$ With photoactivation, donors such as $\mathbf{4}$ and $\mathbf{5}$ have been shown to reduce a wide range of difficult substrates, even including alkylbenzenes. ${ }^{[5,6]}$

In previous studies, it was established that radical cations of neutral donors, e.g. 7 or $\mathbf{1 0}$, which are formed by electron donation to a substrate $\mathrm{RX}$, behave as persistent radicals ${ }^{[7]}$ and combine with radicals, $R^{*}$, derived from the substrate (Scheme 2 ). In the case of TTF 1, the trapping occurs on the sulfur atom of the radical cation 7 to give sulfonium salt $\mathbf{9}$, from which 1 can be regenerated usefully in situ by radical-polar crossover reaction, ${ }^{11]}$ but for the nitrogen-containing radical cations, derived from the super electron donors 3-6, trapping occurs on carbon (e.g. $10 \rightarrow 12)$ and the trapped species 12 is then not available for further useful chemistry. ${ }^{[8]}$ This impedes the use of donors 3-6 in radical chain reactions. In this paper, we provide a solution to this longstanding issue by altering the nature of the super electron donors.

The plan is shown in Scheme 3. Dihydrobenzimidazole 13 was selected as a precursor of the single-electron donor $14{ }^{[9]}$ Initiation by hydrogen atom transfer would afford 14. At any time, species 14 would only be present in trace amounts and could not accumulate since it would be formed as an intermediate in the chain reaction shown. Accordingly, its concentration would be too

[a] S. Rohrbach, Prof. Dr. T. Tuttle, Prof. Dr. J. A. Murphy Dept. of Pure and Applied Chemistry, University of Strathclyde, 295 Cathedral Street, Glasgow G1 1XL, United Kingdom E-mail:John.Murphy@strath.ac.uk

[b] Dr. R. S. Shah

GlaxoSmithKline Medicines Research Centre

Gunnels Wood Road, Stevenage SG1 2NY, United Kingdom Supporting information for this article is given via a link at the end of the document. low to quench substrate-derived radicals effectively. Donor 14 reacts with substrate to form radical $\mathrm{R}^{\circ}$ and the benzimidazolium salt 15 . The radical evolves to radical $R^{2}$ and abstracts $\mathrm{H}$ from 13 to complete the radical chain. Our plans would meanwhile reduce the cation in 15 back to the dihydrobenzimidazole 13 in situ with a mild hydridic reducing agent. Thereby cation 15 would act as an organocatalyst that is converted into an organic super electron donor $\mathbf{1 4}$ during its catalytic turnover.
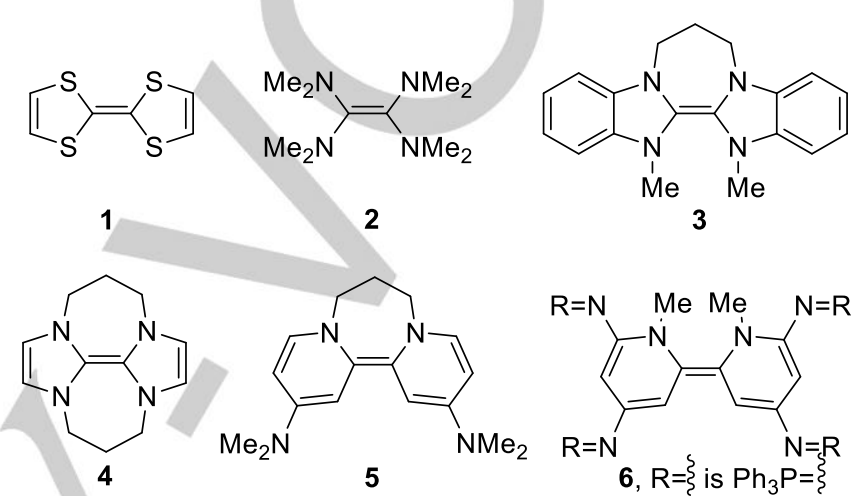

Scheme 1. Organic super electron donors 3-6, and predecessors 1 and 2.

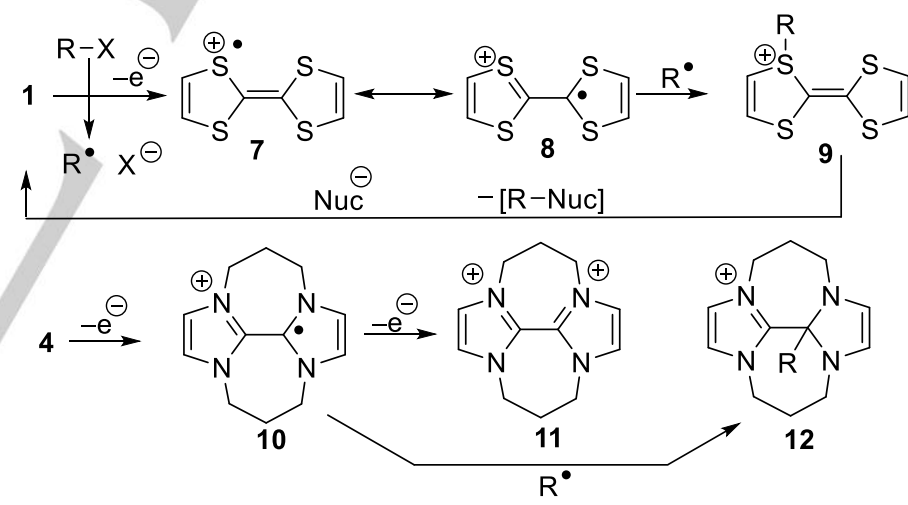

Scheme 2. The established electron donors afford radical cations that readily undergo combination reaction with substrate-derived radicals.

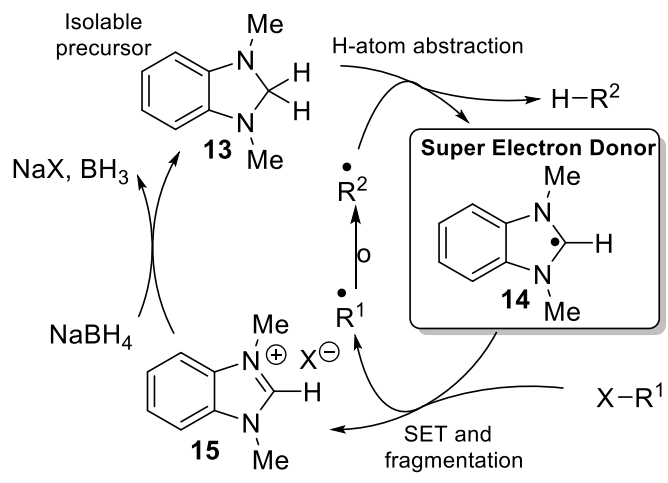

Scheme 3. Proposal for reaction cycle with organic electron donor 14. 
Since little is known ${ }^{[10]}$ about dihydrobenzimidazoles as reducing agents in radical reactions, we firstly investigated the chemistry of 13. Later, the aim would be to investigate how the full catalytic cycle can be closed (Scheme 3).

Compound 13 was obtained in high yield by reacting benzimidazolium salt 15-I with $\mathrm{NaBH}_{4}$ (Scheme 4). ${ }^{[11 \mathrm{a}]}$ The material did not need inert atmosphere or dry conditions, making 13 a convenient precursor of an organic super electron donor. ${ }^{[12]}$

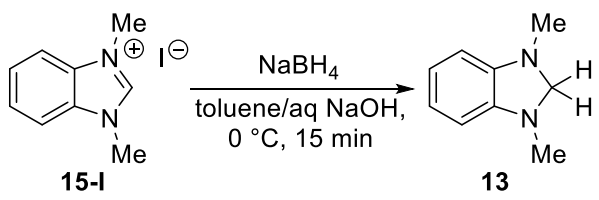

$83 \%$ yield $(20 \mathrm{mmol}$ average of three experiments)

Scheme 4. Synthesis of dihydrobenzimidazole 13

In preliminary optimisation studies, ${ }^{[11 \mathrm{~b}]}$ the reactions were left open to air, and a moderate temperature of $55{ }^{\circ} \mathrm{C}$ gave conveniently high reaction rates. With substrate 16 , a more detailed analysis of the optimal conditions was undertaken (Table 1). Specifically, the effect of dodecanethiol was studied, which acts as a polarity reversal catalyst (PRC). ${ }^{[12]}$ Entry 3 shows that 0.2 equiv. of dodecanethiol enhances the reaction rate and the overall yield; this would arise by mediating the hydrogen abstraction from compound 13 to afford the electron donor $14 . .^{[13]}$ Decreasing [PRC] led to lower yield of $\mathbf{1 7}$ and to longer reaction times (Entries 1 and 2; an equivalent trend was also observed in $\mathrm{MeCN}$ as the solvent $\left.{ }^{[11 \mathrm{c}]}\right)$. Performing the reaction under inert atmosphere $\left(\mathrm{N}_{2}\right.$ or $\left.\mathrm{Ar}\right)$ markedly decreased the reaction rate, supporting our hypothesis that air acts as an initiator (Entry 4). The optimal conditions were then applied to a range of substrates (Scheme 5, Conditions A). The conditions worked well with 5-exotrig reactions involving an unactivated alkene (18a, 18d and 20)

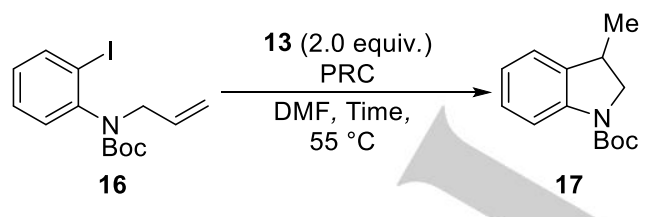

Table 1. Optimisation of a reductive radical cyclisation reaction with $\mathbf{1 3}$

\begin{tabular}{llll}
\hline Entry & PRC ${ }^{[a]}$ & Time $(\mathrm{h}: \mathrm{min})^{[\mathrm{b}]}$ & Yield of 17 \\
\hline 1 & none & $3: 00$ & $64 \%(65 \%)$ \\
2 & $1: 00$ & $76 \%$ \\
3 & 0.05 equiv. & $0: 50$ & $87 \%(86 \%)$ \\
$4^{[\mathrm{d}]}$ & 0.2 equiv. & $6: 00$ & $62 \%[\mathrm{e]}$ \\
\hline
\end{tabular}

[a] Dodecanethiol was used as a polarity reversal catalyst (PRC) [b] The reaction progress was monitored by GC-FID (gas chromatography with flame ionization detection) and the time when the reaction reached full conversion is given. [c] Yields were determined vs an internal standard by GC-FID. The yields of isolated product $\mathbf{1 7}$ are given in brackets. [d] The reaction was performed under inert atmosphere. [e] Remaining starting material (22\%) was also isolated.

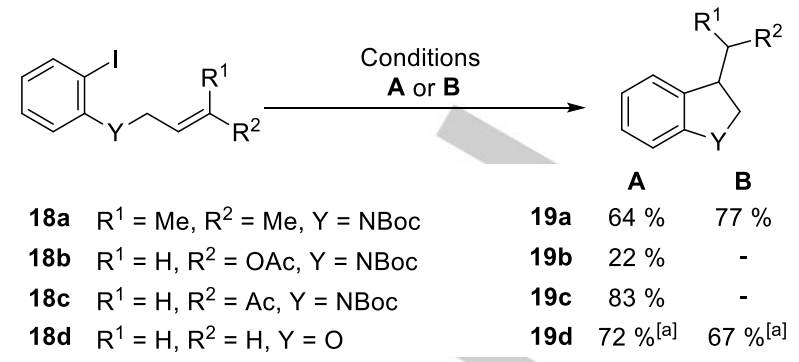

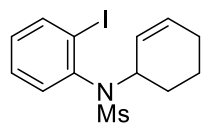
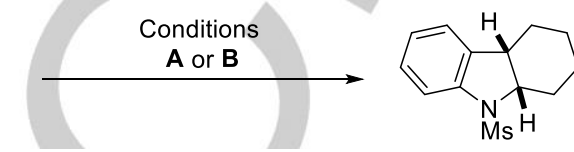

20

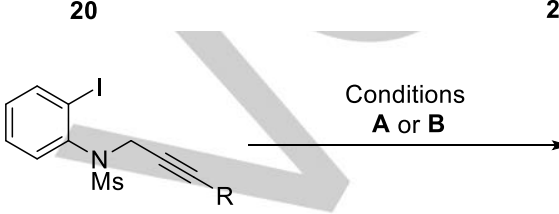

$2187 \% \quad 62 \%$
$R$

22a $\quad \mathrm{R}=\mathrm{Me}$

22b $\quad R=C y$

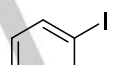

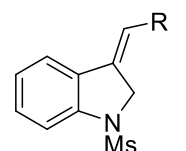

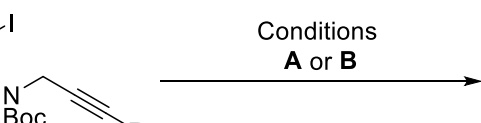

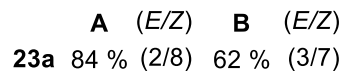

23b $90 \%(3 / 7) \quad 83 \%(2 / 8)$

$\begin{array}{cccc}\text { A } & (E / Z) & \text { B } & (E / Z) \\ 4 \% & (2 / 8) & 62 \% & (3 / 7)\end{array}$

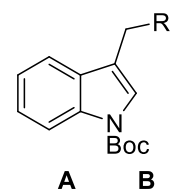

25a $79 \% \quad 53 \%$

24a $\mathrm{R}=\mathrm{CH}_{2}$ OTBDBPS

25b $36 \% \quad 36 \%$ 24b $\mathrm{R}=\mathrm{H}$

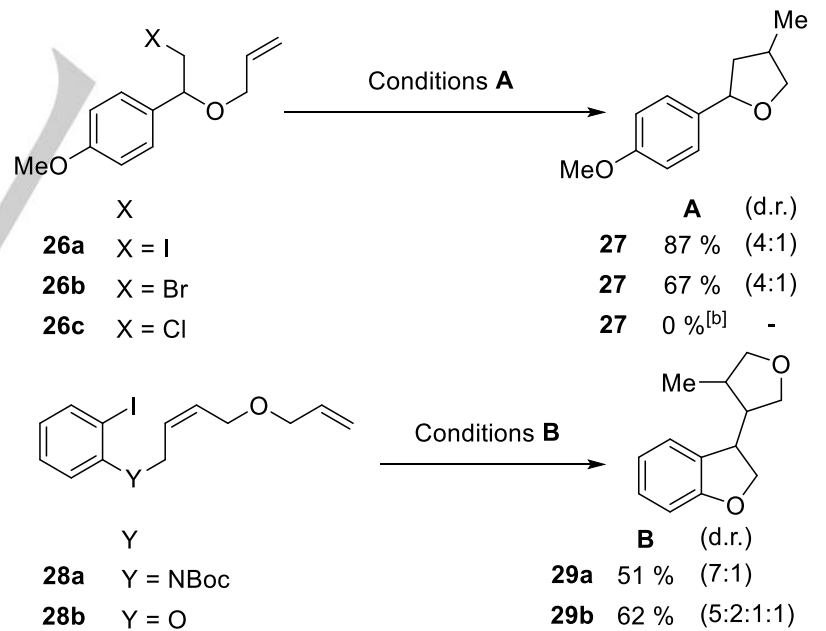

Scheme 5. Substrate scope. Conditions A: Substrate (1.0 equiv.), aminal 13 (2.0 equiv.) dodecanethiol ( 0.2 equiv.), DMF (dimethylformamide, $0.5 \mathrm{M})$, open to air. Conditions B: Substrate (1.0 equiv.), catalyst 15-I (0.2 equiv.) dodecanethiol (0.2 equiv.), $\mathrm{NaBH}_{4}$ (2.0 equiv.), DMF (0.5 M), open to air. [a] Yields were determined by ${ }^{1} \mathrm{H}-\mathrm{NMR}$ vs. an internal standard. [b] directly reduced by-product was isolated in small amounts ${ }^{[8]} ;[\mathrm{c}] 70 \%$ recovered starting material.

or an electron-poor alkene (18c) to give the corresponding cyclised products 19a, (64\%), 19d (83\%), 21 (72\%) and 19c $(87 \%)$ in very good yields. The electron-rich enol-ester $18 \mathrm{~b}$ was less compatible with the protocol and gave only a low yield of cyclised product 19b. 5-Exo-dig cyclisation of the alkynes 22a and 22b gave the indoline products 23a (84\%) and 23b (90\%) in 
excellent yields. Similarly, high yields were obtained for the substrate 24a. In this case the indoline intermediate isomerised to the indole product 25 a $(79 \%)$ during purification. Only the terminal alkyne $\mathbf{2 4 b}$ gave the cyclised product $\mathbf{2 5 b}$ in a low yield. The unactivated alkyl iodide and bromide 26a and 26b were viable substrates, too, and gave product 27 in $87 \%$ yield and $67 \%$ yield, respectively. No product was detected in the reaction of the alkyl chloride $\mathbf{2 6 c}$ and $70 \%$ of the starting material was recovered.

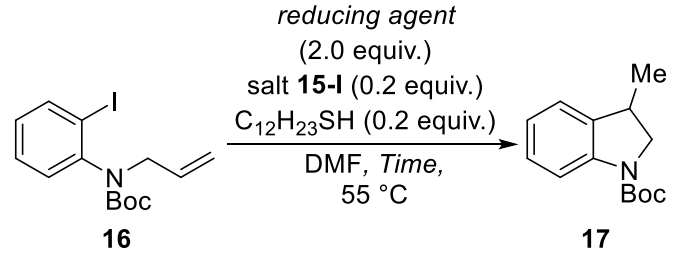

Table 2. Optimisation of the catalytic protocol with salt 15-I.

\begin{tabular}{llll}
\hline Entry & reducing agent & Time $(\mathrm{h}: \mathrm{min})^{[\mathrm{a}]}$ & Yield of 13 $^{[\mathrm{b}]}$ \\
\hline 1 & $\mathrm{NaBH}_{4}$ & $3: 00$ & $84 \%(83 \%)$ \\
2 & $\mathrm{NaBH}(\mathrm{OAc})_{3}$ & $30: 00$ & $35 \%[\mathrm{c]}$ \\
3 & $\mathrm{NaBH}_{3} \mathrm{CN}$ & $20: 00$ & $2 \%{ }^{[\mathrm{d}]}$ \\
$4^{[\mathrm{e}]}$ & $\mathrm{NaBH}_{4}$ & $4: 00$ & $61 \%{ }^{[\mathrm{f}]}$ \\
$5^{[\mathrm{g}]}$ & $\mathrm{NaBH}_{4}$ & $20: 00$ & $9 \%[\mathrm{~h}]$ \\
\hline
\end{tabular}

[a] The reaction progress was monitored by GC-FID and the time when the reaction reached full conversion is given. [b] Yields were determined vs an internal standard by GC-FID. In brackets the yields of pure isolated product 17is given. [c] Remaining starting material $65 \%$. [d] Remaining starting material $98 \%$. [e] 0.05 equiv. of salt 15-I were used. [f] Yield determined by ${ }^{1} \mathrm{H}-\mathrm{NMR}$ vs internal standard. No remaining substrate. [g] Blank reaction in the absence of salt 15-I. [h] Remaining starting materia [87\%] was determined by ${ }^{1} \mathrm{H}-\mathrm{NMR}$ vs internal standard.

To build on these encouraging results, we explored the use of the electron donor $\mathbf{1 4}$ in a catalytic manner. In several of the above reactions with dihydrobenzimidazole 13, the formation of the salt 15-I was observed. ${ }^{[11 b]}$ It was thus natural to address the conversion of this salt back to $\mathbf{1 3}$ in situ with an appropriate terminal reducing agent. Thereby the catalytic cycle would be closed as shown in Scheme 3. Model substrate 16 was again chosen to develop a protocol where the electron donor would be formed catalytically (Table 2). As a starting point, the optimal conditions for reactions with 16 were chosen (Table 1, Entry 3) but 13 was substituted by 15-I ( 0.2 equiv) and. sodium borohydride (2.0 equiv) (Table 2, Entry 1 ). Pleasingly, the product $17(84 \%)$ was formed in almost the same yield as in the reaction with 2.0 equiv. of 13 (87\%) (cf. Table 1, Entry 3). Milder terminal reducing agents than $\mathrm{NaBH}_{4}$, such as $\mathrm{NaBH}(\mathrm{OAc})_{3}$ and $\mathrm{NaBH}_{3} \mathrm{CN}$ gave inferior results (Entry 2 and 3 ). Decreasing the loading of the organocatalyst 15-I from 0.2 . to 0.05 equiv. led to a much lower yield (Entry 4). In a control reaction without the catalyst 15-I, the cyclised product $\mathbf{1 7}$ was only formed in trace amounts (Entry 5).
The catalytic protocol was then applied to substrates in Scheme 5 (Conditions B). The 5-exo-trig cyclisation with 18a, 18d and 20 gave the corresponding cyclized products 19a (77\%), 19d (67\%) and 21 (62\%) in good yields. The substrates 22a, 22b, 24a and $24 \mathrm{~b}$ gave rise to the indolenine products $\mathbf{2 3 a}$ and $\mathbf{2 3 b}$ and indole products $25 \mathrm{a}$ and $25 \mathrm{~b}$. The reactivity that was previously observed with Conditions $A$ was essentially reproduced by the catalytic Conditions B. Finally, we put the catalytic protocol to the test with more complex radical cascade reactions where two carbon-carbon bonds are formed in tandem. From the substrates $\mathbf{2 8 a}$ and $\mathbf{2 8 b}$, the tricyclic products $\mathbf{2 9 a}$ and $\mathbf{2 9 b}$ were obtained in satisfactory yields of $51 \%$ and $62 \%$, respectively. Overall, the results with the catalytic Conditions $B$ demonstrate that it is possible to achieve comparably high yields to the Conditions $A$ which had used 13 in stoichiometric amounts.

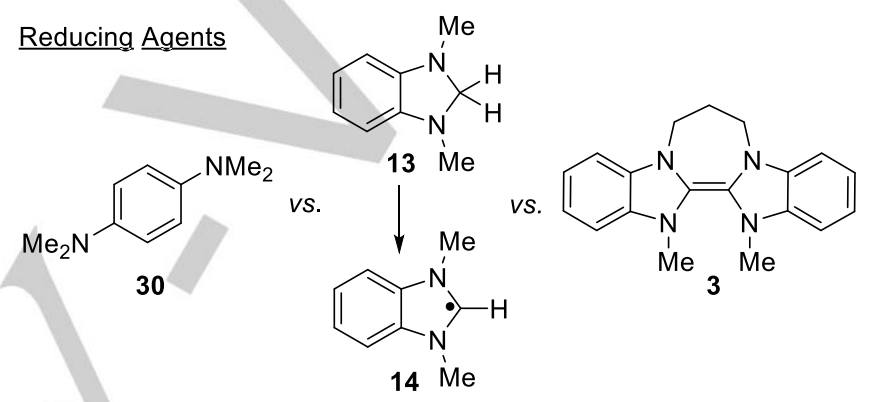

Reaction

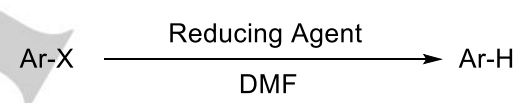

Table 3. Benchmarking the electron donor $\mathbf{1 4}$ against $\mathbf{3 0}$ and $\mathbf{3}$

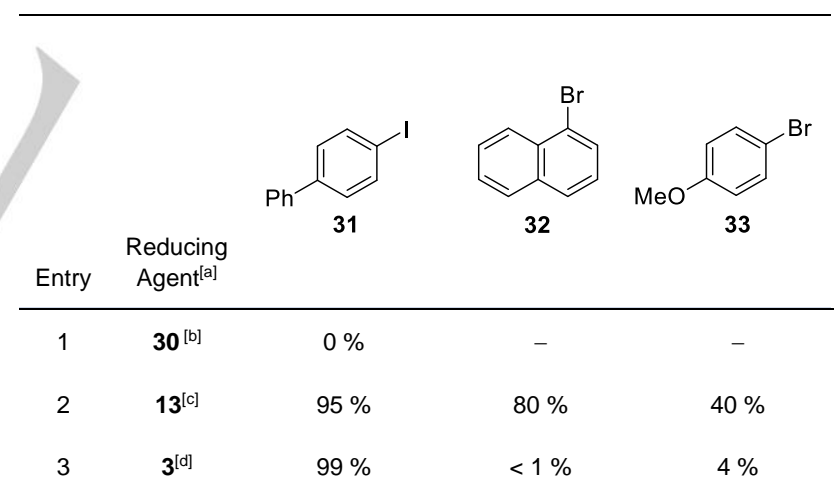

[a] Conversion measured by ${ }^{1} \mathrm{H}-\mathrm{NMR}$. [b] 30 (2.0 equiv.), dodecanethiol (0.2 equiv.), DMF $(0.5 \mathrm{M}), 55{ }^{\circ} \mathrm{C}, 4 \mathrm{~h}$, open to air. [C] 13 (2.0 equiv.), dodecanethiol (0.2 equiv.), DMF $(0.5 \mathrm{M}), 55^{\circ} \mathrm{C}, 4 \mathrm{~h}$, open to air. [d] According to a standard literature procedure: ${ }^{[13]} \mathbf{1}$ (2.0 equiv., formed in situ), DMF $(0.25 \mathrm{M}), 100^{\circ} \mathrm{C}, 18 \mathrm{~h}$, inert atmosphere, sealed tube.

The radical $\mathbf{1 4}$ is structurally related to $\mathbf{3}$. However, the reducing power of $\mathbf{1 4}$ is markedly greater than the reducing power of the parent electron donor 3 . Through computational studies, ${ }^{[11 d]}$ and by cyclic voltammetry experiments, ${ }^{[14]}$ species 14 was found to be more reducing than 3 by approximately $1 \mathrm{~V}$. An analogous observation was made by Giri et al. on their system. ${ }^{[15]}$ In fact, with a reported ${ }^{[14]}$ oxidation potential of $-1.86 \mathrm{~V}$ vs SCE (saturated calomel electrode), electron donor, $\mathbf{1 4}$, is amongst the most potent neutral organic ground state reducing agents known. ${ }^{[3 \mathrm{~d}]}$ 
To assess how this greater reducing power translates into reactivity, we directly compared the reactivity of the single electron donor $\mathbf{1 4}$ with the previously explored electron donor $\mathbf{3}$ (Table 3). Additionally, we sought experimental support that it is actually 14 that acts as a reducing agent in our system and not its closed-shell precursor 13. Phenylenediamine $\mathbf{1 3}$ is electron-rich and might potentially act as an electron donor even without undergoing hydrogen atom abstraction. Compounds $\mathbf{1 3}$ and $\mathbf{3 0}$ are similar in their electronic nature as diamines but $\mathbf{3 0}$ can't give rise to a radical species analogous to 14 (i.e. a radical species where a gain in aromaticity can result from one-electron oxidation) In our hands, $\mathbf{3 0}$ was incapable of reducing even the easiest-toreduce substrate $\mathbf{3 1}$, in the series $\mathbf{3 1 - 3 3}$. This observation substantiates our hypothesis that $\mathbf{1 3}$ does not act as an electron donor in its own right towards this substrate. It needs to be converted to $\mathbf{1 4}$ to give rise to a potent reducing agent. With our optimal conditions (as identified in Table 1, Entry 3), we found that 4-phenyliodobenzene 31 was dehalogenated almost quantitatively. Also, the more difficult to reduce 1bromonaphthalene 32 was reduced in high yield and the even more challenging 4-bromoanisole 33 was reduced in $40 \%$ yield. With the previously established electron donor $3,{ }^{[1 \mathrm{~b}]}$ the aryl bromide substrates $\mathbf{3 2}$ and $\mathbf{3 3}$ could not be reduced even at elevated temperature. Only the aryl iodide substrate $\mathbf{3 1}$ was susceptible to reduction with electron donor 3 . This comparison clearly shows that the new protocol is superior to the protocol with electron donor $\mathbf{3}$ in terms of reducing power.

In conclusion, we have demonstrated that dihydrobenzimidazole 13 is a readily accessible precursor of the potent single electron donor 14. Mild temperatures, fast reaction rates and no need to establish an inert atmosphere are the key characteristics of this protocol. Further, the electron donor 14 can be accessed in a catalytic cycle starting with the salt 15-I. To the best of our knowledge, this is the first example where a neutral organic super electron donor has been used in a catalytic cycle. Viewed from a more general perspective, we have shown how a suitable heterocycle can react with a mild hydridic reducing agent to access a highly reducing intermediate. ${ }^{[16]}$

Further investigations in our laboratory will focus on expanding the principle presented here to other classes of organic electron donors.

\section{Acknowledgements}

We thank the EPSRC UK National Mass Spectrometry Facility at Swansea University for high-resolution mass spectrometry analysis. We thank The University of Strathclyde and GSK for funding.

Keywords: catalysis • organic electron donor • reduction • single electron transfer $\cdot$ upconversion

[1] (a) J. A. Murphy, C. Lampard, N. Lewis, J. Chem. Soc., Chem. Commun., 1993, 295-297. (b) J. A. Murphy, "The Radical-Polar Crossover Reaction", chapter 2.7 in Radicals in Organic Synthesis, Vol. 1, eds.: P. Renaud and M. Sibi, WileyVCH, Germany, 2001.

[2] C. Burkholder; W. R. Dolbier, Jr., M. Médebielle. J. Org. Chem. 1998, 63, 5385-5394. (b) M. Médebielle, W. R. Dolbier, Jr. J. Fluorine Chem., 2008 129, 930-942. (c) M. Mohan, J. A. Murphy, F. LeStrat, H. P. Wessel, Beilstein J. Org. Chem., 2009, 5, 1.
[3] (a) J. A. Murphy, T. A. Khan, S. -Z. Zhou, D. W. Thomson and M. Mahesh Angew. Chem. Int. Ed., 2005, 44, 1356-1360. (b) J. A. Murphy, S.-Z. Zhou, D. W. Thomson, F. Schoenebeck, Mohan M., S. R. Park, T. Tuttle and L. E. A. Berlouis, Angew. Chem. Int. Ed., 2007, 46, 5178-5183. (c) J. A. Murphy, J. Garnier, S. R. Park, F. Schoenebeck, S.-Z. Zhou and A. T. Turner, Org. Lett., 2008, 10,1227-1230 (d) S. S. Hanson, E. Doni, K. T. Traboulsee, G. Coulthard, J. A. Murphy, C. A. Dyker, Angew. Chem. Int. Ed. 2015, 54, 11236-11239. (e) J. D. Martin, C. A. Dyker, Can. J. Chem. 2018, 96, 522-525. (f) S. S. Hanson. N. A. Richard, C. A. Dyker, Chem- Eur J., 2015, 21: 8052-8055.

[4] For reviews, see (a) E. Doni, J. A. Murphy, Chem. Commun. 2014, 50, 6073-6087; (b) J. A. Murphy, J. Org. Chem. 2014, 79, 3731-3746; (c) J. Broggi, T. Terme, P. Vanelle, Angew. Chem. Int. Ed. 2014, 53, 384-413.

[5] (a) E. Cahard, F. Schoenebeck, J. Garnier, S. P. Y. Cutulic, S. Zhou, J. A. Murphy, Angew Chem. Int. Ed., 2012, 51, 3673-3676; (b) E. Doni, B. Mondal, S. O'Sullivan, T. Tuttle, J. A. Murphy, J. Am. Chem. Soc. 2013, 135, 10934-10937. (c) S. O'Sullivan, E. Doni, T. Tuttle, J. A. Murphy, Angew. Chem. Int. Ed. 2014, 53, 474-478.

[6] Organic electron donors are diverse in their structures and application, e.g. (a) G. Tintori, P. Nabokoff, R. Buhaibeh, D. Bergé-Lefranc, S. Redon, J. Broggi, P. Vanelle, Angew. Chem. Int. Ed. 2018, 57, 3148-3153). (b) Q. Wang, M. Poznik, M. Li, P. J. Walsh, J. J. Chruma, Adv. Synth.Catal. 2018, 360, 2854-2868. (c) D. Mandal, R. Dolai, N. Chrysochos, P. Kalita R.Kumar, D. Dhara, A. Maiti, R. S.Narayanan, G. Rajaraman, C. Schulzke, V. Chandrasekhar, A. Jana, Org. Lett. 2017, 19, 5605-5608. (d) M. Brasholz, Angew. Chem. Int. Ed. 2017, 56, 10280-10281. (e) M. Neumann, S. Füldner, B. König, K.Zeitler, Angew. Chem. Int. Ed. 2011 50, 951-954. (f) I. Ghosh, B. König, Angew. Chem. Int. Ed. 2016, 55, 7676-7679. (g) B. Eberle, E. Kaifer, H.-J. Himmel, Angew. Chem. Int. Ed. 2017, 56, 3360-3363. (h) J. Broggi, M. Rollet, J.-L. Clément, G. Canard, T. Terme, D. Gigmes, P. Vanelle, Angew. Chem. Int. Ed. 2016, 55, 59945999. (i) H. Herrmann, M. Reinmuth, S. Wiesner, O. Hübner, E. Kaifer H. Wadepohl, H.-J. Himmel, Eur. J. Inorg. Chem. 2015, 13, 2345-2361.

[7] (a) H. Fischer, J. Am. Chem. Soc. 1986, 108, 3925-3927. (b) A. Studer Chem. Eur. J. 2001, 7, 1159-1164.

[8] J. A. Murphy, F. Schoenebeck, N. J. Findlay, D. W. Thomson, S.-Z. Zhou, J. Garnier, J. Am. Chem. Soc. 2009, 131, 6475-6479.

[9] (a) H. Chikashita, H. Ide, K. Itoh, J. Org. Chem. 1986, 51, 5400-5405. (b) D. D. Tanner, J. J. Chen, J. Org. Chem. 1989, 54, 3842-3846. (c) B. D. Naab, S.Guo, S. Olthof, E. G. B. Evans, P. Wei, G. L. Millhauser, A. Kahn, S. Barlow, S. R. Marder, Z. Bao, J. Am. Chem. Soc. 2013, 135, 15018-15025. (d) For pioneering studies combining $\mathrm{H}$ atom transfer with electron transfer, see R. J. Kolt, D. D. M. Wayner, D. Griller, J. Org. Chem. 1989, 54, 4259-4260.

[10] (a) J. R. Ames, M. A. Houghtaling, D. L. Terrian, T. P. Mitchell, Can. J. Chem. 1997, 75, 28. (b) X.-Q. Zhu, M.-T. Zhang, A. Yu, C.-H. Wang, J.P. Cheng, J. Am. Chem. Soc. 2008, 130, 2501-2516.

[11] (a) For a typical literature procedure see T. Igarashi, E. Tayama, H. Iwamoto, E. Hasegawa, Tetrahedron Lett. 2013, 54, 6874-6877. This procedure was substantially modified to suit our needs, see supporting information. (b) See supporting information, "Biphenyl $\mathbf{3 4}$ - Preliminary Optimisation Studies of Reaction Conditions" p7-p9. (c) See supporting information, "tert-Butyl-3-methylindoline-1-carboxylate 17 - Optimisation for General Procedure A", p9 - p11. (d) See supporting information, "Calculated Oxidation Potentials" p37.

[12] For alternative outcomes of reactions of imidazolium salts with $\mathrm{NaBH}_{4}$, see (a) S. Gardner, T. Kawamoto, D. P. Curran, J. Org. Chem. 2015, 80, 9794-9797; (b) E. F. Godefroi, J. Org. Chem. 1968, 33, 860-862.

[13] The aminal hydrogen atoms in $\mathbf{1 3}$ are hydridic. Consequently, the abstraction of one of these hydrogen atoms by a nucleophilic carbon centred radical is slow due to a mismatch in polarity. The thiol acts as a mediator in this step. The hydrogen abstraction from the thiol by a carbon centred radical is matched in polarity and fast. The thiyl radical that is generated is electrophilic and can efficiently abstract an aminal hydrogen atom. For further information on PRC see (a) B. P. Roberts, Chem. Soc. Rev. 1999, 28, 25-35; (b) H. S. Dang, M. R. J. Elsegood, K.-M. Kim, B. P. Roberts, J. Chem. Soc., Perkin Trans. 1 1999, 2061-2068; (c) A. J. Fielding, B. P. Roberts, Tetrahedron Lett. 2001, 42, 4061-4064. 
[14] (a) X.-Q. Zhu, M.-T. Zhang, A. Yu, C.-H. Wang, J.-P. Cheng, J. Am Chem. Soc. 2008, 130, 2501. (b) J. R. Aranzaes, M.-C. Daniel, D. Astruc Can. J. Chem. 2006, 84, 288

[15] G. N. Reddy, S. Giri, Phys. Chem. Chem. Phys. 2016, 18, 24356-24360.
[16] M. A. Syroeshkin, F. Kuriakose, E. A. Saverina, V. A. Timofeeva, M. P. Egorov, I. Alabugin, Angew. Chem. Int. Ed. 2019, 58, 5532-5550. 
1

Entry for the Table of Contents (Please choose one layout)

\section{COMMUNICATION}

This paper reports an organocatalytic role for a benzimidazolium salt in radical chemistry. A highly reducing intermediate $(-1.86 \mathrm{~V}$ vs. SCE) is produced simply by treatment with $\mathrm{NaBH}_{4}$ and then using air as initiator. This is the first time that an organic super electron donor has been used catalytically. and introduces a novel catalytic approach for the upconversion of reducing power.

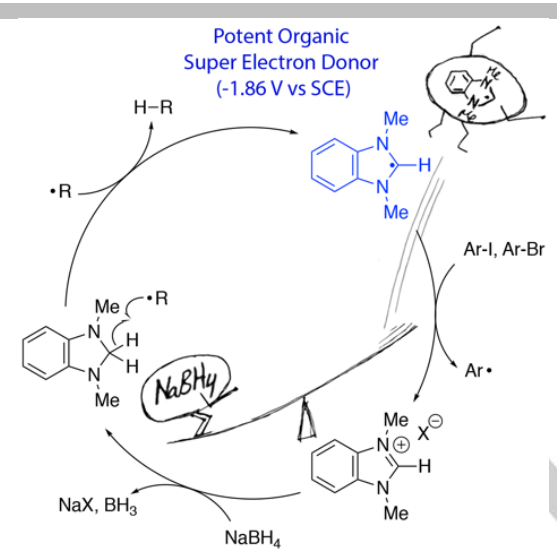

S. Rohrbach, R. S. Shah, T. Tuttle, * and J. A. Murphy*

Page No. - Page No.

CatalySED! Neutral Organic Super Electron Donors Made Catalytic 
Click here to access/download Supporting Information SI_CatalyticDonor_submit 100519.pdf 